


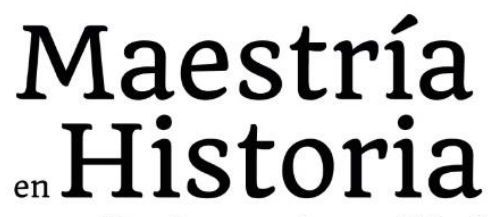

Acreditada en Alta Calidad

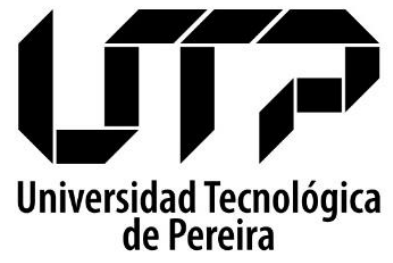

ESTUDIOS HISTÓRICOS

LAS PRÁCTICAS DIDÁCTICAS DE LOS MAESTROS DE CIENCIAS SOCIALES Y SU INCIDENCIA EN EL INTERÉS DE LOS ESTUDIANTES POR EL ÁREA EN CUATRO INSTITUCIONES EDUCATIVAS DE SANTIAGO DE CALI

THE DIDACTIC PRACTICES OF SOCIAL SCIENCES TEACHERS AND ITS IMPACT ON THE INTEREST OF STUDENTS IN THE AREA IN FOUR EDUCATIONAL INSTITUTIONS OF SANTIAGO DE CALI

Luis Bernardo Betancur Cruz pp. 25-45

DOI: https://doi.org/10.22517/25392662.21851

Vol. 4 Núm. 2 | Julio-diciembre de 2020

Pereira, Colombia

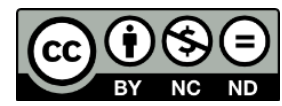




\title{
LAS PRÁCTICAS didÁCTICAS de LOS MAESTROS DE CIENCIAS SOCIALES Y SU INCIDENCIA EN EL INTERÉS DE LOS ESTUDIANTES POR EL ÁREA EN CUATRO INSTITUCIONES educativas de Santiago de Cali*
}

\author{
THE DIDACTIC PRACTICES OF SOCIAL SCIENCES TEACHERS AND ITS IMPACT ON THE INTEREST \\ OF STUDENTS IN THE AREA IN FOUR EDUCATIONAL INSTITUTIONS OF SANTIAGO DE CALI
}

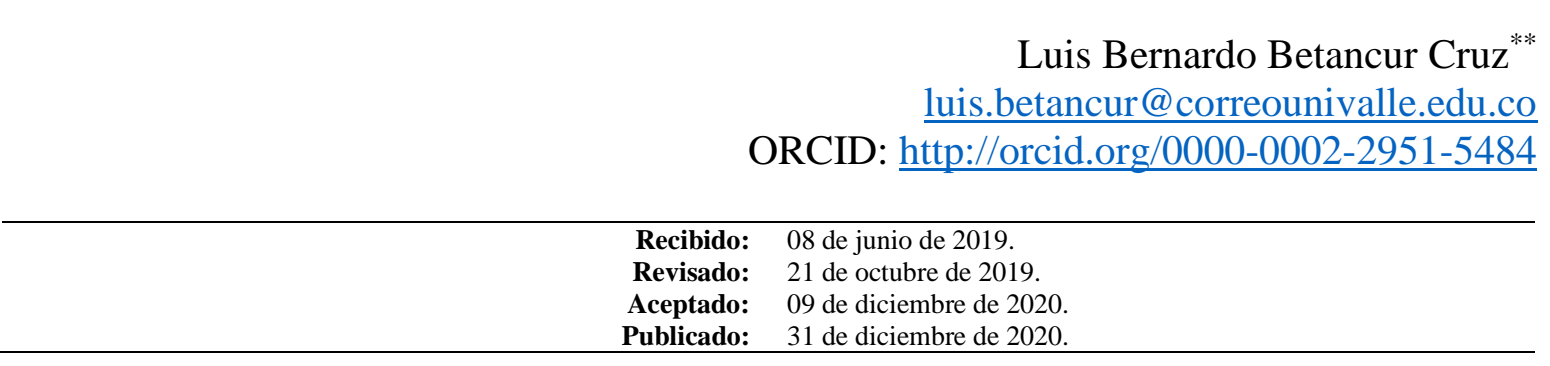

\section{Resumen}

El artículo expone las percepciones que tienen de las prácticas didácticas tanto estudiantes como maestros de Ciencias Sociales de cuatro instituciones educativas de la ciudad de Cali. A partir del contraste de percepciones, se pretende analizar la incidencia que pueden tener las prácticas didácticas en el interés por el área, y su potencial relevancia en la formación del pensamiento social e histórico del estudiante. El método utilizado fue el mixto; las muestras se recogieron a partir de dos encuestas, una aplicada a 10 profesores y otra a 661 estudiantes de grado 6.o, 9.o y 11.0 en las cuatro instituciones educativas. El texto concluye que la enseñanza centrada en la trasmisión de contenidos es promotora del desinterés y que es necesario aprovechar los métodos de la investigación social e histórica para transitar hacia metodologías de enseñanza que promuevan la movilización de pensamiento social e histórico.

Palabras clave: Didáctica, didáctica de las ciencias sociales, maestro, estudiante, prácticas didácticas.

\begin{abstract}
The article exposes the perceptions that both students and teachers of Social Sciences of four Educational Institutions of the city of Cali have of the didactic practices. From the contrast of perceptions, it is intended to analyze the impact that didactic practices may have on the interest in the area and its potential relevance in the formation of social and historical thought in the student. The method used was the mixed one, the samples were collected from two surveys, one applied to 10 teachers and another to 6616 th, 9th, and 11th grade students in the four educational institutions. The text concludes that teaching focused on the transmission of content is a promoter of disinterest and that it is necessary to take advantage of the methods of social and historical research to move towards teaching methodologies that promote the mobilization of social and historical thought.
\end{abstract}

Key Words: Didactics, didactics of the social sciences, teacher, student, teaching practices.

\footnotetext{
* Este documento respeta las directrices y normas dispuestas en la Declaración de Ética de Publicación de Ciencia Nueva, Revista de Historia y Política. Esta declaración puede consultarse en la página web de la revista: http://revistas.utp.edu.co/index.php/historia

${ }^{* *}$ Licenciado en Historia, Universidad del Valle. Magíster en Historia, Universidad del Valle. Profesor de la Facultad de Educación, Universidad Santiago de Cali. Profesor del Departamento de Historia, Universidad del Valle.
} 


\section{Introducción}

El texto pretende interrogar por las prácticas didácticas de los maestros de ciencias sociales $^{1}$, contrastando la manera en cómo los agentes implicados en el proceso de la enseñanza-aprendizaje (profesor y estudiante) subjetivan el proceso mismo de la enseñanza. Es decir, tanto profesores como estudiantes exponen una imagen o representación de lo que ha caracterizado la forma de enseñar ciencias sociales y la manera como circula el conocimiento enseñado.

Teóricamente la investigación se aproxima a la discusión sobre la didáctica de las ciencias sociales, a partir de la reflexión sobre las prácticas de enseñanza en las cuatro instituciones educativas trabajadas. Esta discusión tiene como base la definición de didáctica acotada por Armando Zambrano, quien entiende la didáctica como reflexión y acción sobre la enseñanza «su objeto de estudio [refiriéndose a la didáctica] es la génesis, circulación y apropiación del saber y sus condiciones prácticas de enseñanza y aprendizaje» ${ }^{2}$. La génesis como la condición innata por conocer, que se ha acumulado en el tiempo y configurado como un saber sobre las cosas que explican el mundo, que le permite al maestro erigirlo en la práctica desde la circulación (discurso, estrategias y herramientas). En esta investigación, la etapa de apropiación no se piensa desde el contenido que se aprende, sino desde la funcionalidad individual y social que tiene el conocimiento aprendido. La investigación acude a la práctica para mirar los principios didácticos que define el maestro para desarrollar dicha práctica. Otros conceptos articuladores serán el de conciencia histórica y pensamiento histórico desarrollados por Jorn Rüsen ${ }^{3}$.

El trabajo de investigación utilizó metodología mixta. Según James MacMillan, entre las características que precisan la investigación cualitativa están la definición de variables o categorías de análisis, que puedan analizarse a la luz de información cualitativa o estadística ${ }^{4}$; es decir que, aunque la información primaria sea meramente cuantitativa, las variables permiten tener información que posibilita el análisis cualitativo. El diseño de los instrumentos estuvo supeditado a cuatro categorías de análisis: 1) el profesor de Ciencias Sociales y sus principios pedagógicos para el trabajo en el aula, 2) el profesor y su trabajo didáctico, 3) estrategias y herramientas usadas para la enseñanza de las ciencias sociales y 4) formas de evaluación. El desarrollo de la primera categoría dependió de la información brindada por los maestros, las tres categorías restantes dependieron de la información recolectada con los estudiantes.

Se buscó tanto desde el diseño y aplicación del instrumento, así como a partir de la construcción del documento, establecer una correlación entre la categoría 1 y las categorías

\footnotetext{
${ }^{1}$ Joan Pagès hace, a mi juicio, una definición clara de ciencias sociales escolares a partir de su enseñanza: «En la mayoría de países que utilizan en la escuela obligatoria la expresión "enseñanza de las ciencias sociales" se sigue haciendo fundamentalmente una enseñanza disciplinar centrada, en primer lugar, en la enseñanza de la historia, en segundo lugar en la enseñanza de la geografía - las dos disciplinas tradicionales en nuestro currículo-, en la historia del arte - la mayor parte de las veces integrada a historia-, en la educación o formación cívica o para la ciudadanía y a mucha distancia, y con carácter muy minoritario y casi anecdótico, en otras disciplinas sociales separadamente o con enfoques interdisciplinares» J. Pagès, «Enseñar y aprender ciencias sociales en el siglo XXI: reflexiones casi al final de una década» (Investigación en Educación, Pedagogía y Formación Docente, II Congreso Internacional, Medellín, 2009), 2.

${ }_{2}^{2}$ A. Zambrano. Didáctica, pedagogía y saber (Bogotá: Cooperativa Editorial Magisterio, 2005), 21.

3 J. Rüsen, Razãao histórica: Teoría da historia, fundamentos da ciência histórica (Brasilia: Editora Universidad de Brasilia, 2001).

${ }^{4}$ J. McMillan, Investigación Educativa (Madrid: Pearson Educación S.A, 2005).
} 
2, 3 y 4, con el objetivo de constituir una relación causa y efecto entre las prácticas didácticas de los maestros de Ciencias Sociales y la incidencia de estas en el interés de los estudiantes por las ciencias sociales. Se tomó como base para analizar la influencia en el interés, la percepción de los estudiantes sobre la misma práctica del maestro (información que se concretó desde las variables 2, 3 y 4). Cabe resaltar que la investigación no se centró solamente en instituciones educativas públicas, pretendiendo acercar una pequeña mirada a la enseñanza de las ciencias sociales desde una institución privada. Sin embargo, si es coincidente el tipo de población que atiende en términos de estratos socioeconómicos, condición que también logra dar muestras de coincidencias en los principios didácticos de los maestros y las representaciones de los estudiantes.

Los grados en los que se recolectó la información para el desarrollo de la investigación fueron $6 .^{\circ}, 9 .^{\circ}$ y $11 .^{\circ}$. La razón por la que no se tomaron todos los grados fue por el amplio volumen de información, el tiempo del que se disponía para desarrollar el proceso de investigación y los costos. De esta manera, el grado $6 .^{\circ}$ representa el inicio de la educación básica secundaria, el grado $9 .^{\circ}$ corresponde a la etapa intermedia de la educación básica secundaria y el grado $11 .^{\circ}$ corresponde al último grado de la básica secundaria, pero también de lo que se denomina media vocacional que atañe a los grados $10 .^{\circ}$ y $11 .^{\circ}$.

El trabajo se realizó en cuatro instituciones educativas de la ciudad de Cali, tres de ellas son de carácter público y atienden población escolar de diferentes estratos, tabla 1.

Tabla 1. Instituciones educativas encuestadas, ubicación y estratificación de la población estudiantil

\begin{tabular}{|c|c|c|}
\hline Institución educativa - IE & Ubicación & $\begin{array}{c}\text { Estratificación población } \\
\text { estudiantil }\end{array}$ \\
\hline $\begin{array}{c}\text { Institución Educativa Santa } \\
\text { Librada }\end{array}$ & Barrio San Bosco & 1,2 y 3 \\
\hline $\begin{array}{c}\text { Institución Educativa Luz } \\
\text { Haydee Guerrero Molina }\end{array}$ & Barrio Los Robles & 1,2 y 3 \\
\hline $\begin{array}{c}\text { Institución Educativa } \\
\text { Rodrigo Lloreda }\end{array}$ & $\begin{array}{c}\text { Corregimiento el Saladito, } \\
\text { Cali }\end{array}$ & 1,2 y 3 \\
\hline Liceo Napolitano & Barrio el Troncal & 2,3 y 4 \\
\hline
\end{tabular}

Fuente: elaboración propia.

Cuando se pensó en las instituciones que podían servir de referencia para desarrollar la investigación, se tuvieron en cuenta dos aspectos necesarios que le darían peso a la estructura argumentativa del documento. Primero, tener una base de información primaria amplia que, apoyada con la discusión que ha suscitado la enseñanza de las ciencias sociales en Colombia, lograra dar elementos para pensar la realidad de la enseñanza de las ciencias sociales escolares, al menos en la ciudad. Segundo, la ubicación de las instituciones educativas, y en el caso de las IE públicas, que fueran sedes centrales, pues son estas las que albergan mayor número de población escolar. Las cuatro instituciones educativas se encuentran situadas en zonas estratégicas para el acceso de población escolar de diferentes comunas e incluso de la

\footnotetext{
${ }^{5}$ De aquí en adelante se acotará la sigla IE para referirse a institución educativa.
} 
ciudad en general, como es el caso de Santa Librada, ubicada en el centro de la ciudad y que por tradición y tamaño es una IE a la que muchos padres buscan que sus hijos ingresen. La IE Luz Haydee Guerrero acoge escolares de buena parte de la franja oriental de la ciudad, también conocida como distrito de Aguablanca ${ }^{6}$. La IE Rodrigo Lloreda ubicada en la zona rural de $\mathrm{Cali}^{7}$, exactamente en la vía al mar, corregimiento el Saladito, es la IE más grande de la zona y recibe población escolar de corregimientos como Felidia, la Elvira e incluso del Barrio Terrón Colorado. Finalmente, el Liceo Napolitano, ubicado en el centro oriente de la ciudad, y sin ser una IE pública, alberga escolares de estratos 2 y 3 , en ella confluyen estudiantes de las comunas 8, 11 y 12. A continuación un mapa que muestra las zonas de influencia que tienen las instituciones educativas en las que se realizó la investigación.
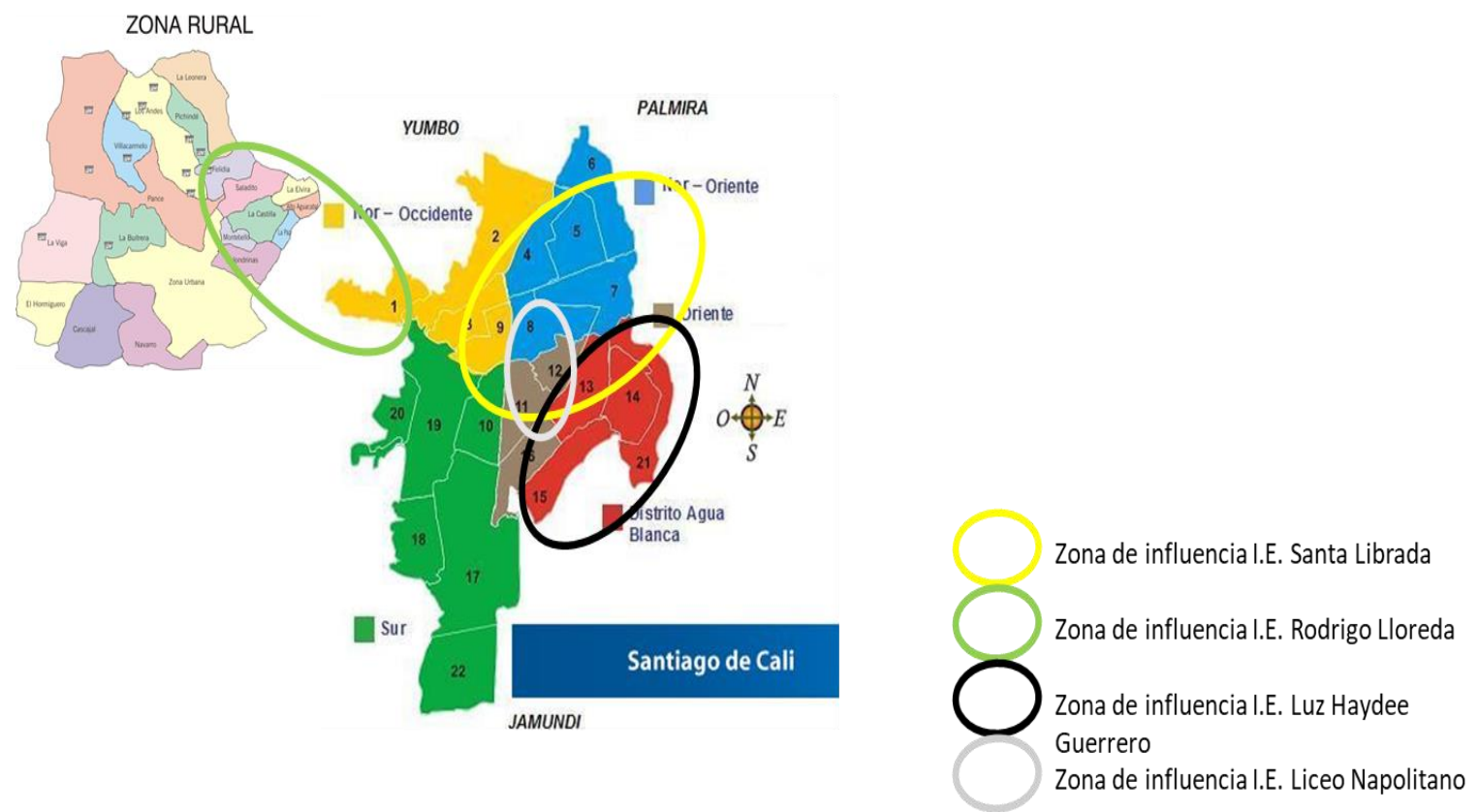

Mapa 1. Zonas de cooptación escolar de las instituciones educativas encuestadas, 2017

Fuente: Alcaldía de Santiago de Cali, 2017. Elaboración propia.

Las encuestas se aplicaron a un total de 661 estudiantes de grado $6 .^{\circ}, 9^{\circ}$ y $11 .^{\circ}$, discriminados de la siguiente manera:

Tabla 2. Discriminación de la población estudiantil encuestada según grado y grupos

\footnotetext{
${ }^{6}$ El Distrito de Aguablanca está ubicado al oriente de la ciudad de Cali, está conformado por las comunas 13, 14, 15 y 21, alberga una población aproximada de 850.000 habitantes, según datos del Censo de 2005.

${ }^{7}$ Dentro de la investigación se consideró una I.E rural con el propósito de tener una mirada del tipo de enseñanza de las ciencias sociales que se está trabajando en este contexto, y poder tener al menos a pequeña escala una lectura de la percepción que tienen los escolares que viven en contextos rurales de las ciencias sociales que les enseñan.
} 


\begin{tabular}{|c|c|c|c|}
\hline $\begin{array}{c}\text { Institución } \\
\text { educativa }\end{array}$ & $\begin{array}{l}\text { Estudiantes } \\
\text { encuestados }\end{array}$ & Grado & Grupos por grado \\
\hline \multirow[t]{3}{*}{ IE Santa Librada } & \multirow[t]{3}{*}{217} & $6 .^{\circ}$ & 5 \\
\hline & & $9 .^{\circ}$ & 3 \\
\hline & & $11 .^{\circ}$ & 3 \\
\hline \multirow{3}{*}{$\begin{array}{l}\text { IE Luz Haydee } \\
\text { Guerrero Molina }\end{array}$} & \multirow[t]{3}{*}{167} & $6 .^{\circ}$ & 4 \\
\hline & & $9 .^{\circ}$ & 2 \\
\hline & & $11 .^{\circ}$ & 1 \\
\hline \multirow[t]{3}{*}{ IE Rodrigo Lloreda } & \multirow[t]{3}{*}{206} & $6 .^{\circ}$ & 3 \\
\hline & & $9 .^{\circ}$ & 2 \\
\hline & & $11 .^{\circ}$ & 3 \\
\hline \multirow[t]{3}{*}{ Liceo Napolitano } & \multirow[t]{3}{*}{71} & $6 .^{\circ}$ & 1 \\
\hline & & $9 .^{\circ}$ & 1 \\
\hline & & $11 .^{\circ}$ & 1 \\
\hline Totales & 661 & 12 & 29 \\
\hline
\end{tabular}

Fuente: encuesta a estudiantes grados $6 .^{\circ}, 9^{\circ}$ y $11 .^{\circ}$. Elaboración propia, 2017.

Por otra parte, se aplicó una encuesta a un total de 10 profesores en las cuatro IE. Este número de profesores obedece a quienes están a cargo de la enseñanza de las ciencias sociales en los grados $6 .^{\circ}, 9^{\circ}$ y $11 .^{\circ}$, discriminados de la siguiente manera:

Tabla 3. Número de docentes de Ciencias Sociales encuestados por institución educativa

\begin{tabular}{|c|c|}
\hline Institución educativa & Número de profesores encuestados \\
\hline IE Santa Librada & 3 \\
\hline IE Rodrigo Lloreda & 4 \\
\hline IE Luz Haydee Guerrero Molina & 2 \\
\hline Liceo Napolitano & 1 \\
\hline Total & 10 \\
\hline
\end{tabular}

Fuente: encuesta a profesores de Ciencias Sociales. Elaboración propia, 2017.

a. El profesor de Ciencias Sociales y sus principios pedagógicos para el trabajo en el aula

Cuando se decidió explorar la trascendencia que tienen las prácticas de enseñanza desarrolladas por el maestro, partíamos de una hipótesis que había emanado de la experiencia a través de la observación en campo y como resultado de preocupaciones en el orden epistemológico y curricular de la formación de licenciados en ciencias sociales; pero también, de aquellas reflexiones que han venido suscitándose en el ámbito académico desde hace unas tres décadas en Iberoamérica, donde se han cuestionado, entre otras cosas, las formas de enseñanza de las ciencias sociales en el ámbito escolar. Por tal razón, en este apartado se explora, a partir de las prácticas, los principios pedagógicos que determinan las formas de 
trabajo en el aula, dichos principios tienen que ver con las orientaciones pedagógicas que están detrás del trabajo en clase desarrollado por el profesor.

Para Pagès y Santisteban la enseñanza de las ciencias sociales se ha caracterizado por estar centrada en la transmisión directa de información predefinida y soportada sobre currículos rígidos cargados de contenidos, que hacen del estudiante un sujeto receptor pasivo. Este tipo de enseñanza brinda datos que le pueden servir al estudiante para ampliar su conocimiento en cultural general, pero no le provee herramientas cognitivas, praxiológicas y socio afectivas para comprender y enfrentar el presente, así como para proyectar el futuro ${ }^{8}$. Para Pagès son dos los problemas que perviven y que no permiten que se avance hacia una enseñanza de las ciencias sociales que prepare para enfrentar las situaciones de la vida:

En primer lugar, la pervivencia y la resistencia de un currículum nacionalista y eurocéntrico útil para el control de la ciudadanía. En segundo lugar, la pervivencia de un currículum cargado de contenidos factuales centrados sobre los hechos y los períodos más relevantes de cada país y del pasado europeo ${ }^{9}$.

Históricamente el oficio del maestro ha estado supeditado a la trasmisión de conocimiento científico académicamente acumulado, por lo menos desde el momento en que el pensamiento ilustrado europeo triunfó definitivamente sobre las bases políticas y escolásticas que habían sembrado cientos de años atrás las monarquías y la iglesia católica. Saldarriaga llamaba la atención a este asunto refiriéndose a los primeros destellos del pensamiento pedagógico moderno hacia los siglos XVI y XVII, y cómo este más adelante en el surgimiento de los sistemas educativos republicanos se convirtió en un instrumento que terminó por legitimar de asiento la función socializadora y reguladora de la escuela.

Las epistemologías científicas que han gobernado al saber pedagógico desde su nacimiento moderno, en los siglos XVI y XVII, con los humanistas del renacimiento (Vives, Erasmo, Ramus, Comenio) hasta los refinamientos experimentales de los siglos XIX y XX, han puesto a la escuela y al maestro en el trabajo de iniciar a niños y jóvenes en el camino ascendente y cada vez más selectivo de la ciencia y para ello el saber pedagógico ha sido diseñado como un repertorio de técnicas para reproducir, en la escuela, los procedimientos de los diversos tipos de disciplinas científicas ${ }^{10}$.

Así, para Saldarriaga, el maestro ha sido un iniciador, alguien en quien recae la potestad de encaminar a los estudiantes por el sendero del conocimiento, es el portador legítimo del saber, clase a clase, a partir de los rudimentos de ciencia que expone frente al tablero. Esto quiere decir que en el maestro recae una fuerza histórica que se mantiene hasta la actualidad desde una enseñanza que sigue centrada en la trasmisión de contenidos, reafirmando lo que dice Pagès respecto a las razones que hacen de la enseñanza de las ciencias sociales un ejercicio procedimental y repetitivo que no afecta positivamente en la formación de pensamiento crítico y reflexivo en el estudiante.

Si bien en las últimas décadas ha habido cambios respecto a los fines y formas de la enseñanza de las ciencias sociales, estos no han sido lo suficientemente significativos como

\footnotetext{
8 J. Pagès, «Ciudadanía global y enseñanza de las Ciencias Sociales: retos y posibilidades para el futuro», Revista de Investigación en Didáctica de las Ciencias Sociales, REIDICS, n. ${ }^{\circ} 5$ (2019): 5-22, doi: https://doi.org/10.17398/2531-0968.05.5 A. Santisteban, «Las finalidades de la enseñanza de las Ciencias Sociales», en Didáctica del conocimiento del medio social y cultural en la educación primaria, ed. por J. Pagès y A. Santisteban (Madrid: Editorial Síntesis, 2016).

${ }^{9}$ Pagès, «Ciudadanía global y enseñanza de las Ciencias Sociales: retos y posibilidades para el futuro», 8.

10 O. Saldarriaga, «Del oficio de maestro ¿de intelectual subordinado a experto subordinador?», Revista Educación y Ciudad, IDEP, n. ${ }^{\circ} 11$ (2006): 53-70.
} 
para transformar definitivamente las prácticas didácticas de los maestros y maestras. En el caso de Colombia, y particularmente el área de Historia que hace parte del conjunto de disciplinas de las ciencias sociales y que ha cumplido un papel importante dentro de la asignatura de Ciencias Sociales en la escuela, desde finales de la década de $1970^{11}$ se intentó revalorizar el conocimiento histórico desde una nueva propuesta de contenidos que iban acordes a los desarrollos en nuestro país de la disciplina histórica, y que empezaban a hacer presencia a través de una nueva camada de historiadores que, habiendo bebido de la Escuela francesa de los Anales y los marxistas británicos, colocaban en cuestión la historia que vanagloriaba los héroes de la patria y los acontecimientos que había dado pie a la formación de la nación colombiana, historia que se había promovido desde la Academia Colombiana de Historia y que se había enseñado en las escuelas del país a través de manuales escolares.

Por otra parte, el movimiento pedagógico en la década de 1980, y académicos como el profesor Darío Betancourt y Renán Vega ${ }^{12}$ en la década de 1990 llamaron la atención ante el imperceptible adelanto en la enseñanza de Historia, porque a pesar de la reforma curricular de 1984 y de los avances en la disciplina histórica se seguía enseñando una historia oficial cargada de sentimiento nacionalista; sus métodos seguían siendo el dictado y sus herramientas el tablero, el cuaderno y el libro de texto ${ }^{13}$.

A continuación, una serie de datos que muestran, para el caso de cuatro instituciones educativas en Cali, el contraste entre la continuidad, expresada en prácticas de enseñanza tradicionales, que a decir de Pagès:

transmite informaciones pero que crea pocas condiciones para que el alumnado las haga suyas, se las apropie desarrollando un pensamiento personal, crítico. Una enseñanza demasiado a menudo basada en supuestas verdades, en la que la realidad expresada en el currículo y en los textos se confunde con la realidad que está fuera de ellos. En la que el pasado se confunde con su interpretación. En la que el presente se confunde con lo que uno ve o con lo que le cuentan a través de los medios. Una enseñanza acrítica, un aprendizaje poco transferible a la realidad ${ }^{14}$.

Y el cambio, expresado en la búsqueda y aplicación de formas alternativas de enseñanza que promueven en el estudiante la movilización de ideas y recursos para la formación de pensamiento crítico y reflexivo, a decir de Pagès:

\footnotetext{
${ }^{11}$ Encabezado por Jaime Jaramillo Uribe y Jorge Orlando Melo, se presentó en los años 70, a solicitud del Ministerio de Educación Nacional, una propuesta para reformar los contenidos que se enseñaban en historia; en este informe se proponía «al MEN orientar una nueva propuesta de estructura curricular para la enseñanza de la Historia de Colombia» al MEN orientar una nueva propuesta de estructura curricular para la enseñanza de la Historia de Colombia. L.B. Betancur, «A propósito de Bauman y los retos de la educación en la modernidad liquida: cavilaciones sobre el futuro de la enseñanza de la Historia», en El prisma de la formación docente en Colombia: teoría pedagógica y experiencias didácticas, ed. por C. Cano (Cali: Editorial Universidad Santiago de Cali, 2017), 44.

${ }^{12}$ C. Guerrero, «La incidencia de las reformas educativas en la enseñanza de la historia en Colombia, 19732007» (tesis de maestría, Universidad Nacional de Colombia, Bogotá, 2011).

${ }^{13}$ S. Mosquera, G. Villa y A. M. Riascos, «Interrelación de los sistemas (político, económico, educativo y religioso) y su afectación en el subsistema de la enseñanza de la historia de Colombia en el periodo de 1900 a 1958» (trabajo de grado, Universidad Santiago de Cali, Cali, 2017). El manual de historia de José María Henao y Hernando Arrubla de 1910, como señalan las autoras del trabajo de grado en mención, continuó siendo empleado como uno de los libros de texto que guiaba la enseñanza de la historia colombiana en las instituciones educativas, en donde se identificó su uso en los planes de curso de la IE de Santa Librada para casi todo el siglo $\mathrm{XX}$.

${ }^{14}$ J. Pagès, «Ciudadanía global y enseñanza de las Ciencias Sociales: retos y posibilidades para el futuro», 14.
} 
En definitiva, repensar las finalidades y adecuar los contenidos [de las ciencias sociales ${ }^{15}$ ] acercándolos a la realidad parece que puede facilitar unos aprendizajes mucho más significativos que aquel conocimiento escolar histórico, geográfico y social que se limitaba a describir cómo era nuestra nación, cómo se había forjado y quiénes habían sido los padres de la misma ${ }^{16}$.

Se realizó una encuesta a los maestros de Ciencias Sociales de las cuatro instituciones educativas, trabajadas para indagar por los principios que definen su trabajo pedagógico en el aula. Luego de analizada la información, se concluyó que hay una permanencia de formas tradicionales de enseñanza en las prácticas de los maestros; en un $60 \%$, el discurso oral, el tablero y el libro de texto son las herramientas más usadas para enseñar Ciencias Sociales en el aula, alternando casi que paralelamente con el uso de herramientas audiovisuales (por ejemplo, el video beam), en un $30 \%$. Estas cifras también muestran que tienen cabida en un espacio considerable las formas visuales de trabajo pedagógico que posibilitan una enseñanza más amena, menos monótona y que les permite a los estudiantes formarse una idea del tema trabajado a partir de las imágenes, aunque la enseñanza se centre en la transmisión de contenidos, no en problematizarlos ${ }^{17}$.

En contraste a esta información, a la pregunta por los mediadores más utilizados para enseñar Ciencias Sociales, todos los profesores encuestados coinciden en que el discurso narrativo apoyado con material escrito y la orientación en clase son los más comunes y adecuados para trabajar en el aula, coincidiendo con lo dicho en el párrafo anterior donde una mayoría de profesores $(60 \%)$ se identifica con las formas tradicionales de enseñanza.

Por otra parte, a pesar de que los datos muestran que es una preocupación para los profesores que el estudiante pueda asociar las imágenes mostradas a través de medios audiovisuales con las temáticas abordadas, el cambio de herramienta no significa un cambio en la función que cumple el conocimiento en la formación del estudiante. Ya lo referenciaba Betancur en una investigación previa sobre prácticas de enseñanza en Guadalajara de Buga: «Las TIC resultan ser herramientas que complementan el trabajo realizado con las tecnologías tradicionales y se ajustan, cuando es necesario y conveniente, al modelo de trabajo de profesor» $^{18}$.

Ahora bien, resulta paradójico que los maestros hablen de formar por competencias y de trabajar con base en modelos constructivistas cuando en un alto porcentaje sus herramientas didácticas son el discurso, el tablero y el libro de texto ${ }^{19}$. Esta consideración si tenemos en cuenta que el desarrollo de una competencia ${ }^{20}$ en el estudiante se logra cuando puede utilizar el conocimiento aprendido en una situación de la vida diaria ${ }^{21}$; según los resultados de la encuesta, los maestros de muestran que la formación por competencias en

\footnotetext{
${ }^{15}$ La cursiva es mía.

${ }^{16}$ J. Pagès, «Enseñar y aprender ciencias sociales en el siglo XXI: reflexiones casi al final de una década», 8.

${ }^{17}$ Estaríamos hablando de que continúa anquilosada en la práctica de los maestros la propuesta decroliana, que aproxima a la objetividad de las cosas a partir del acercamiento que pueda tener el individuo a la realidad desde la imagen.

${ }^{18}$ L. B. Betancur, «Una mirada a las prácticas de enseñanza de las ciencias sociales en Guadalajara de Buga», Revista de investigaciones. Universidad Católica de Manizales 16, n. ${ }^{\circ} 2$ (2016), 34.

${ }^{19}$ Esta información será corroborada más adelante a través de lo percibido por los estudiantes.

${ }^{20}$ Llevar a cabo una formación por competencias depende de la capacidad que tiene el maestro para movilizar recursos que le permitan al estudiante aprender y saber utilizar lo aprendido en diferentes situaciones de la vida. M. Ruiz, «La competencia estratégica del docente ante la violencia escolar en países en vías de desarrollo». Revista Interuniversitaria de Formación del Profesorado n. 45 (2002): 169-182.

${ }^{21}$ J. Sacristán, «Diez tesis sobre la aparente utilidad de las competencias», en Educar por competencias ¿Qué hay de nuevo?, ed. por J. Sacristán (Madrid: Ediciones Morata, 2008).
} 
Ciencias Sociales se desarrolla en un $45 \%$ desde la concepción constructivista del aprendizaje y en un $44 \%$ en enfoques afines, como el aprendizaje significativo.

$\mathrm{Si}$ partimos de los datos expuestos, la encuesta a los maestros develaría una discordancia entre el discurso hegemónico de las competencias y su materialización desde modelos constructivistas, pues desde el uso de herramientas y mediadores tradicionales no es posible la experimentación y menos la proposición y la resolución de problemas; esto sin sumarle que un mínimo porcentaje de los maestros realizan actividades académicas por fuera del aula que estimulen un acercamiento experimental a las ciencias sociales. Según la encuesta, solo los maestros de la Institución Educativa Santa Librada promueven en un 100 $\%$ las salidas de campo como estrategia de aprendizaje para acercar de manera distinta a los estudiantes al conocimiento de las ciencias sociales. Estas salidas se discriminaron de la siguiente manera: el $60 \%$ del trabajo se hace a partir de visitas al Archivo Histórico de Cali, un $20 \%$ se desarrolla a través de visitas al Centro Histórico de la ciudad y el restante $20 \%$ se trabaja desde salidas de observación geográfica.

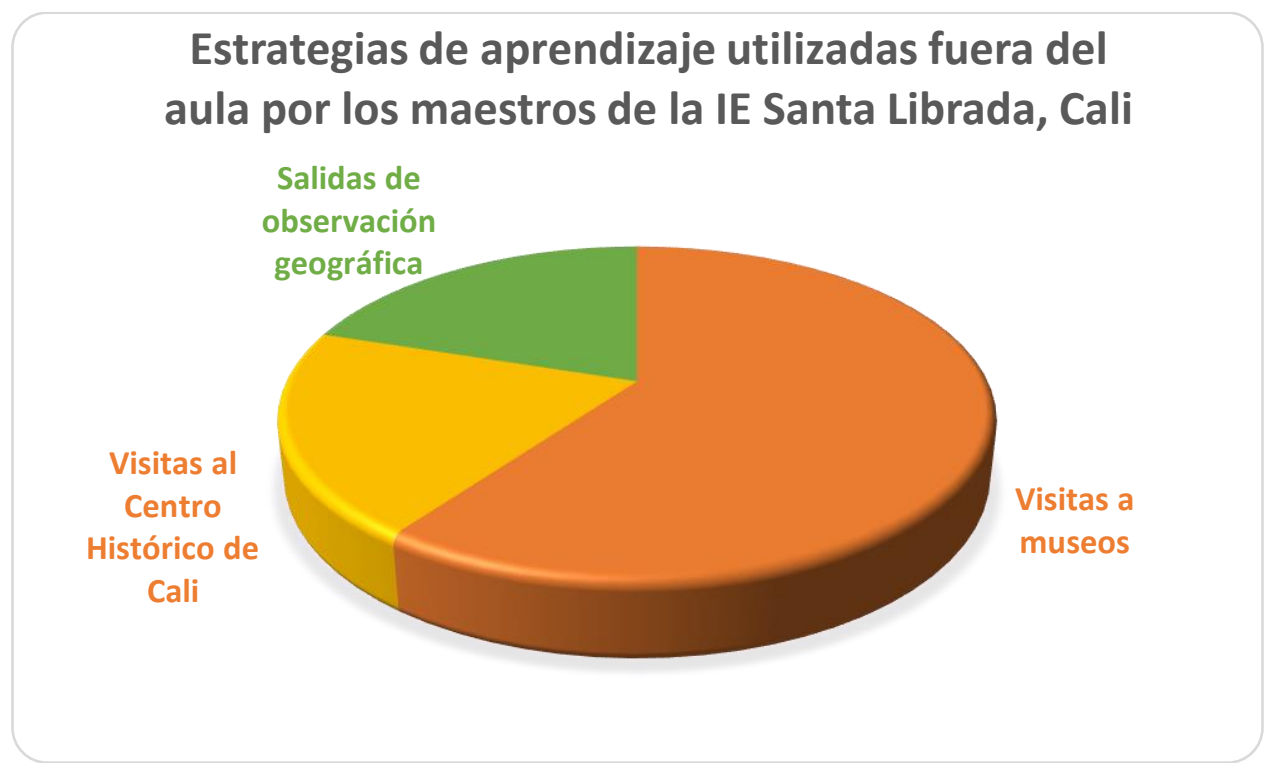

Figura 1. Resultados de las estrategias de aprendizaje en la IE Santa Librada

Fuente: encuesta a profesores de Ciencias Sociales. Elaboración propia, 2017.

A diferencia de ellos, los maestros de la Institución Educativa Luz Haydee Guerrero Molina, en un $75 \%$ señalan no utilizar salidas de campo, el $25 \%$ restante que corresponde a la opción «otras estrategias», refiere a la utilización de estrategias adheridas directamente al trabajo en clase, lo que constata que casi la totalidad del proceso formativo en ciencias sociales no sale del aula. El profesor del Colegio Liceo Napolitano señala utilizar, como recurso alternativo al trabajo en el aula, únicamente las salidas de observación geográfica, sin acudir a otras posibilidades en cuanto a salidas de campo; por ejemplo, visitas al Archivo Histórico o a museos. Mientras que un $60 \%$ de los profesores de la Institución Educativa Santa Librada utilizan como recurso de salida de campo la visita a museos, siendo este recurso el más frecuente de todos, el $40 \%$ restante divide honores entre visitas al Centro Histórico y salidas de observación geográfica. 
Lo anteriormente descrito reafirma una de las reflexiones expuestas en el estudio sobre las prácticas de enseñanza en Buga que realicé en 2016, donde se deja por sentado las limitaciones que tiene centralizar la enseñanza de las ciencias sociales en la exposición oral dentro del salón de clase.

En el campo de las Ciencias Sociales, ciertas categorías conceptuales además de abstractas, revierten complejidad para ser comprendidas desde una mera explicación verbal; resulta difícil para el educando poder comprenderlo, lo que dificultará en él la comprensión del fenómeno social e histórico estudiado $^{22}$.

En este primer apartado se concluye que la enseñanza de las ciencias sociales debe seguir avanzado en el uso de materiales que le permitan al estudiante hacerse una imagen de la realidad social e histórica, no solo desde la magistralidad, porque esta ha creado una percepción en los estudiantes de que Ciencias Sociales es una asignatura que enseña datos, irrelevantes después de verlos en clase. Voss, Wiley y Kennet señalan:

La comprensión de los novatos acerca de la historia quizá esté basada en estereotipos, textos o simplificaciones, una concepción "de hechos, textos y personalidades" de la historia, además, debido al modo en que se enseña historia, los estudiantes pueden percibirla como una mera crónica de acontecimientos $^{23}$.

b. El estudiante y sus consideraciones frente a las prácticas de enseñanza del profesor de Ciencias Sociales: capacidad didáctica, estrategias, herramientas y evaluación

En contraste con lo expuesto por los maestros, la percepción de los estudiantes constituye una mirada desde la vivencia diaria en el aula. Para indagar por las percepciones que tienen los estudiantes de básica secundaria de las prácticas de enseñanza de los maestros de Ciencias Sociales, fue importante examinar las imágenes que han construido sobre las prácticas de enseñanza de sus maestros a lo largo de su experiencia vital en la escuela. Los escenarios propuestos en el instrumento para investigar la imagen que los estudiantes tenían de las prácticas de enseñanza del profesor de Ciencias Sociales fueron tres: 1) el profesor y su capacidad didáctica, 2) las estrategias y las herramientas usadas para enseñar y 3) las formas de evaluación.

El interés por analizar los datos a la luz de estos escenarios pretende, en el primer escenario, conocer la manera como el profesor busca trascender los principios didácticos que han orientado históricamente una enseñanza de las ciencias sociales centrada en la trasmisión directa de contenidos por medio del marcador, el tablero y el libro de texto, hacia formas más alternativas. En el segundo, identificar los tipos de herramientas y estrategias pedagógicas utilizadas y la manera en cómo estas guardan relación con el tipo de enseñanza. Finalmente, identificar y analizar cómo se evalúa el proceso de enseñanza y la relación que llega a tener, por ejemplo, con los principios didácticos rectores y la naturaleza de las estrategias y las herramientas pedagógicas utilizadas.

${ }^{22}$ Betancur, L.B. «Una mirada a las prácticas de enseñanza de las ciencias sociales en Guadalajara de Buga», 38.

${ }^{23}$ J.F. Voss, J. Wiley y J. Kennet, «Las percepciones de los estudiantes acerca de la historia y los conceptos históricos», en Aprender y pensar la historia, ed. por M. Carretero y J. Voss (Buenos Aires: Amorrortu, 2004), 296. 
Alrededor del primer escenario, para el caso de Colombia, es preciso aclarar que antes del paso a la asignatura de Ciencias Sociales, luego de la promulgación del Decreto 1002 de 1984, se enseñaba en las escuelas Historia y Geografía como áreas independientes ${ }^{24}$; sin embargo, la poca claridad por parte de Ministerio de Educación Nacional sobre que debía enseñarse en Ciencias Sociales, y la ínfima formación que tuvieron los maestros respecto a la enseñanza de la nueva área, significó agrupar dos asignaturas antiguamente independientes junto a un interés por fortalecer la formación cívica en una nueva asignatura ${ }^{25}$.

Sin claridad, la enseñanza de las ciencias sociales dejó abierto hasta comienzos del siglo XXI los contenidos y los procesos de enseñanza a las directrices dadas por los textos escolares y al manejo que el profesor quisiera darle. Con la promulgación de los estándares básicos de competencias en 2004 se centralizó el horizonte disciplinar del área; no obstante, actualmente, al menos en las IE trabajadas en la investigación, los cambios en la forma en cómo se desarrollan las clases siguen mostrando en un alto porcentaje una enseñanza centrada en contenidos y no necesariamente en el desarrollo de competencias ${ }^{26}$.

El análisis de la información recolectada evidencia en un porcentaje promedio del $49.2 \%$ (en el total de las cuatro IE) que, para los estudiantes, el profesor de Ciencias Sociales desarrolla sus clases propiciando espacios para la investigación a partir de preguntas e interrogantes. Promover el interrogante en el caso particular de la enseñanza de la historia, es para Santiesteban una forma propicia para acercar el pasado a la realidad presente ${ }^{27}$. Desde la propuesta de las cuestiones socialmente vivas de Nicole Tutiaux, el interrogante es la base inicial para trabajar aspectos como el interés y la motivación del estudiante, principios fundamentales según este autor para la comprensión de procesos sociales e históricos ${ }^{28}$.

El porcentaje restante, es decir, el $50.8 \%$ indica que los estudiantes consideran que el profesor se dedica a trasmitir contenidos a partir de dos estrategias distintas; el (24.3\%) referencia que la trasmisión es vertical o de manera directa, y el $(26.5 \%)$ señala que trasmiten contenidos relacionándolos con situaciones que viven cotidianamente los estudiantes; en otras palabras, a pesar de establecer una relación entre lo que se enseñanza y la búsqueda de la comprensión por parte del estudiante, la enseñanza sigue centrada en trasmitir contenidos.

\footnotetext{
${ }^{24}$ M. González, «La configuración histórica del saber pedagógico para la enseñanza de la historia en Colombia, trazos de un camino: 1870-2010» (tesis de maestría, 2011), https://www.javeriana.edu.co/biblos/tesis/csociales/tesis249.pdf

${ }^{25}$ Particularmente será la Ley General de Educación la que exprese, aún más, la ambigüedad respecto a qué son y qué debe enseñarse en el área de ciencias sociales; el artículo 23 de la Ley cuando se refiere a los grupos de áreas obligatorias separa historia, geografía, democracia y constitución política de las ciencias sociales. «Artículo 23. Áreas obligatorias y fundamentales. Para el logro de los objetivos de la educación básica se establecen áreas obligatorias y fundamentales del conocimiento y de la formación que necesariamente se tendrán que ofrecer de acuerdo con el currículo y el Proyecto Educativo Institucional. Los grupos de áreas obligatorias y fundamentales que comprenderán un mínimo del $80 \%$ del plan de estudios, son los siguientes: [...] 2. Ciencias sociales, historia, geografía, constitución política y democracia [...]». Congreso de la República de Colombia, Ley 115 del 8 de febrero de 1994, ley general de educación.

${ }^{26}$ González, «La configuración histórica del saber pedagógico para la enseñanza de la historia en Colombia, trazos de un camino: 1870-2010».

${ }^{27}$ A. Santisteban, «La enseñanza de las Ciencias Sociales a partir de problemas sociales o temas controvertidos: estado de la cuestión y resultados de una investigación», El Futuro del Pasado 10 (2019): 57-79, doi: http://dx.doi.org/10.14516/fdp.2019.010.001.002

${ }^{28}$ N. Tutiaux, «Les qüestions socialment vives, un repte per a la Història i la Geografia escolars», en Les qüestions socialment vives i l'ensenyament de les ciències socials, ed. por J. Pagès y A. Santisteban (Barcelona: Universidad Autónoma de Barcelona, 2011).
} 
Los datos del análisis de las preguntas sobre los profesores se pueden desglosar en los tres grados objeto de estudio desde las siguientes gráficas:

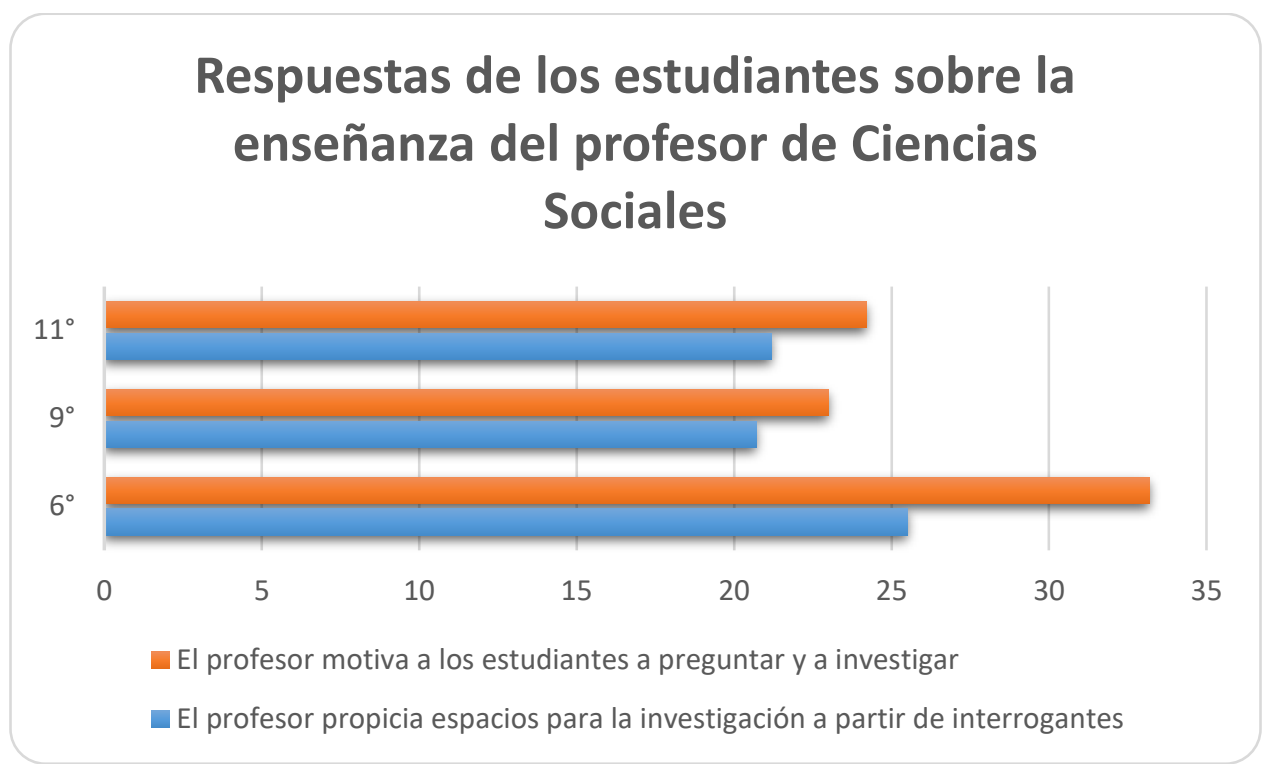

Figura 2. Análisis de resultados a la encuesta de estudiantes de las cuatro instituciones educativas

Fuente: encuesta a estudiantes de las instituciones educativas. Elaboración propia, 2017.



Figura 3. Análisis de los resultados del tipo de contenido transmitidos por los profesores, según los estudiantes de las IE

Fuente: encuesta a estudiantes de las instituciones educativas. Elaboración propia, 2017. 
Asociado al ejercicio de trasmisión directa, la importancia que adquiere el discurso oral es muy alta, puesto que las ciencias sociales son un campo de saber que difícilmente se puede escenificar, materializar o experimentar dentro del aula de clase y resulta ser poco tangible para el estudiante. Aunque el área integra varios campos disciplinares, estos tienen naturalezas distintas y por lo tanto la posibilidad de hacerlo perceptible para el estudiante en el acto pedagógico dependerá de la naturaleza del saber de cada campo y de la pericia del maestro.

En el caso particular de la historia resulta complejo recrear el pasado solo desde una acción retórica o de habla, más cuando para comprenderlo se requiere que el estudiante no solo conozca sobre la temática o, como denomina Seixas, conceptos de primer orden, sino que también desarrolle habilidad para la comprensión de procesos históricos a partir de nociones como tiempo histórico, cambio, permanencia, progreso, declive, causa y efecto, referido también por Seixas como conceptos de segundo orden ${ }^{29}$.

Frente a lo anterior y ante las posibles limitantes didácticas que pueda tener el profesor, enseñar se reduce a un mero acto de trasmisión que deriva en ser un ejercicio discursivo acompañado de un lenguaje verbal ${ }^{30}$, siendo el recurso más común para el maestro hablar prolongadamente. Resultado de la exposición verbal se espera que el estudiante pueda hacer una elaboración mental del proceso social e histórico y con ello logre dar cuenta de la realidad explicada; de esta manera se revalida que un notable porcentaje de maestros reproducen las lógicas de la escolarización clásica. Saldarriaga, Quiceno y Ghotme han reiterado desde sus investigaciones que la escuela ha sido un espacio de incorporación al orden social, y por lo tanto el conocimiento enseñado por trasmisión directa a yacido como un instrumento regulador que permite coaccionar al individuo desde la acción disciplinaria ${ }^{31}$. Otros como Carretero acusan a los métodos memorísticos como los artífices de una visión de la historia cuyo objetivo ha sido la de forjar identidades de corte nacionalista, reproduciendo consigo los intereses de los grupos políticos de poder ${ }^{32}$.

El segundo escenario tiene que ver con las estrategias y herramientas que el profesor utiliza para la enseñanza de las ciencias sociales. Al indagar por la frecuencia con la que se utilizan diversos tipos de estrategias y herramientas se encontró que el maestro parte de un saber acumulado que es trasmitido a los estudiantes y del que se espera den cuenta, generalmente, a través de talleres individuales y grupales. El uso de esta estrategia es común a lo largo de la secundaria; no obstante, la intensidad con las que se utiliza en clase varía de acuerdo con el grado. La praxis o ejecución de la enseñanza hace parte de los ejes fundamentales que definen la didáctica ${ }^{33}$, llevar a cabo la praxis implica los recursos y los espacios con que cuenta el maestro para constituir un ambiente de aprendizaje, recursos y espacios se ven materializados a través de las herramientas y estrategias utilizadas para mediar en el proceso de enseñanza.

${ }^{29}$ P. Seixas y C. Peck, «Teaching historical thinking», en Challenges and Prospects for Canadian Social Studies, ed. por A. Sears y I. Wright ( Vancouver: Pacific Educational Press, 2004), 109-117.

${ }^{30}$ G. Sanz, Comunicación efectiva en el aula (España: Editorial Grao, 2005).

${ }^{31}$ Saldarriaga, O. «Del oficio de maestro ¿de intelectual subordinado a experto subordinador?». H. Quiceno, Crónicas históricas de la educación en Colombia (Bogotá: Cooperativa Editorial Magisterio, 2003). R. Ghotme, «La identidad nacional, el sistema educativo y la historia en Colombia 1910-1962», Revista Científica General José María Córdova 11, n. 11 (2013): 273-289.

${ }^{32}$ M. Carretero, Documentos de identidad: la construcción de la memoria histórica en un mundo global (Buenos Aires: Paidós, 2007).

${ }^{33}$ A. Zambrano, Didáctica, pedagogía y saber. B. Buitrago, «La didáctica: un acontecimiento vivo en el aula», Revista Científica Guillermo de Ockham 6, n. 2 (2008). 
El uso de estrategias como el taller y el trabajo escrito son más frecuentes a medida que avanza la escolaridad hacia el final de la secundaria, este modelo se invierte y empiezan a ser más recurrentes estrategias como las discusiones en grupo, los foros, la lectura en clase y las mesas redondas en los grados superiores, grado $9 .^{\circ}$ y grado $11 .^{\circ}$; es decir, existe una tendencia que inicia en $6 .^{\circ}$ con las estrategias clásicas de reproducción de saberes (talleres), y cambia en los últimos años de la secundaria (grado $9 .^{\circ}$ y $11 .^{\circ}$ ) hacia estrategias que promueven la discusión, el debate y la proposición de ideas. Aunque en los grados superiores son continuos los talleres grupales e individuales, en comparación a los porcentajes arrojados en grado $6 .^{\circ}$ la diferencia es notable, así como el uso de estrategias que promueven el uso de la palabra por encima del documento escrito.

Paralelamente, el uso de herramientas en el aula de clase es consecuente al tipo de estrategias utilizadas, en los grados $6 .^{\circ}$ las herramientas más frecuentes son el tablero y el marcador, los libros de texto, las guías y fotocopias, mientras que herramientas que promueven el debate como el caso de los documentales son usados de manera poco frecuente; la balanza se equilibra en grado $9 .^{\circ}$ y $11 .^{\circ}$ en la medida que el uso de herramientas, como las películas y los documentales hacen contrapeso a la hegemonía del taller y el trabajo escrito; si bien prevalece según lo referenciado por los estudiantes en un porcentaje del $67 \%$, es evidente una tendencia al uso de herramientas más alternativas a partir de grado $9 .^{\circ}$.

Lo anteriormente expuesto revela al menos dos cosas. La primera, que la formación del estudiante sigue estando centrada en la trasmisión de contenidos, y que son estos un vehículo irreductible para la reproducción de información que se repite año tras año. Segundo, así como los procesos de enseñanza son progresivos, es decir, se parte de trasmitir un contenido simple para ir avanzando hacia contenidos más complejos, así mismo el desarrollo de habilidades comunicativas y habilidades para la comprensión también son progresivas. En los grados $6 .^{\circ}$ se trabaja hacia el desarrollo de habilidades de lectoescritura, esto es evidente en el uso constante de estrategias como el taller y herramientas como el texto escolar o la guía, para luego trabajar habilidades como la interpretación, la proposición, el diálogo y la comunicación asertiva (grados $9 .^{\circ}$ y $11 .^{\circ}$ ) con estrategias como el debate y el foro a partir del uso de películas y documentales.

Esta tendencia resulta ser contradictoria en sí misma en la medida que las estrategias y herramientas que se utilizan en los primeros años de la secundaria no preparan para la comprensión y se trata más bien de fortalecer la lectoescritura a partir de un ejercicio mecánico ${ }^{34}$, pues no exigen la movilización de recursos que abran paso al desarrollo de habilidades metacognitivas; consecuencia de ello, cuando los estudiantes llegan a grados superiores y se les exige trabajar desde estrategias como el debate, la plenaria o el foro se les dificulta elaborar procesos de interpretación.

Finalmente, frente al tercer escenario que refiere a los procesos evaluativos, resulta ser funcional la capacidad de desarrollo didáctico del profesor y las estrategias y herramientas usadas en el proceso de enseñanza dentro del proceso evaluativo; esto es, se constituye una especie de triangulación entre la enseñanza centrada en la trasmisión de contenidos, el uso de tablero y marcador, así como el trabajo con libros de texto y guías, para finalmente dar parte de lo aprendido a través de una evaluación escrita u oral; en este sentido, el maestro espera que el estudiante dé cuenta de lo que a él o ella le han enseñado y con esto sobre entender que el educando ha aprendido.

${ }^{34}$ J. Arboleda, Competencias pedagógicas: conceptos y estrategias (Bogotá: Editorial REDIPE, 2011). 
Cabe resaltar que la evaluación es un proceso subjetivo donde confluyen los intereses del profesor, de la institución educativa y del sistema educativo; todos estos agentes esperan una respuesta del estudiante, respuesta que genera en el educando una profunda sensación de incertidumbre ${ }^{35}$, pues queda expuesto a la subjetivación del maestro, quien tradicionalmente resuelve a favor de las necesidades del saber; este último no termina respondiendo a las necesidades del educando, sino que resulta ser un instrumento del poder coaccionador del maestro $^{36}$ y de otros entes supranacionales llámese BID, FMI, OCDE ${ }^{37}$, mediante pruebas como Saber 11 y PISA $^{38}$.

Revisada y analizada la información arrojada durante el trabajo de campo, se evidenció que el modelo de evaluación que prevalece es la escrita, forma que por lo general pretende dar cuenta sobre lo enseñado al finalizar cada proceso de enseñanza. Según los estudiantes de las cuatro instituciones educativas (del total de los grados $6 .^{\circ}, 9 .^{\circ}$ y $11 .^{\circ}$ ), el $42 \%$ considera que los profesores utilizan la evaluación escrita como mecanismo de seguimiento al proceso de enseñanza llevado a cabo con ellos, mientras el $10.2 \%$ señala que también se utiliza la evaluación oral. Así las cosas, con un porcentaje del 52.7 \% la evaluación tradicional es para los estudiantes la forma más convencional de dar cuenta del conocimiento aprendido. A continuación, los porcentajes por grado en las cuatro instituciones educativas.

Tabla 4. Porcentaje de evaluación escrita según estudiantes de grado $6 .^{\circ}, 9 .^{\circ}$ y $11 .^{\circ}$

\begin{tabular}{|c|c|c|c|}
\hline $\begin{array}{l}\text { Institución } \\
\text { Educativa }\end{array}$ & $\begin{array}{c}\text { Grado 6. } \\
(\%)\end{array}$ & $\begin{array}{c}\text { Grado } 9 .^{\circ} \\
(\%)\end{array}$ & $\begin{array}{c}\text { Grado } 11.0 \\
(\%)\end{array}$ \\
\hline IE Rodrigo Lloreda & 51 & 41 & 6 \\
\hline IE Santa Librada & 27 & 27 & 16 \\
\hline $\begin{array}{l}\text { IE Luz Haydee } \\
\text { Guerrero Molina }\end{array}$ & 49 & 83 & 63 \\
\hline Liceo Napolitano & 43 & 56 & 43 \\
\hline Total \% por grado & 42.5 & 51.7 & 32 \\
\hline & & Total \% & 42 \\
\hline
\end{tabular}

El porcentaje de evaluación escrita, según lo señalado por los estudiantes, evidencia que es todavía muy importante para los maestros verificar lo aprendido al final del proceso de enseñanza de un contenido.

Modelos de evaluación más alternativa, como por ejemplo el seguimiento al proceso de enseñanza a partir de actividades periódicas y retroalimentadas, corresponde, según los estudiantes, solamente al $27.7 \%$, si bien es una cifra considerable en comparación al uso de

\footnotetext{
${ }^{35}$ G. Avanzini, «La incertidumbre de la evaluación», en La pedagogía hoy, ed. por G. Avanzini (México: Fondo de Cultura Económica, 1998).

${ }^{36}$ O. Saldarriaga, «Del oficio de maestro ¿de intelectual subordinado a experto subordinador?».

37 Organizaciones como el Banco Interamericano de Desarrollo, el Fondo Monetario Internacional y la Organización para la Cooperación y el desarrollo Económico son agencias internacionales de orden económico que bajo el influjo del modelo neoliberal inciden directamente en las decisiones de los países en vías de desarrollo para que promuevan políticas que favorecen el crecimiento económico por encima del bienestar social.

${ }^{38}$ M. Foucault, Microfísica del poder (Madrid: Ediciones de la Piqueta, 1979).
} 
la evaluación escrita, la supera en casi el doble, notándose que se realiza con mucha más frecuencia. Reafirmando la idea de que se requiere dar cuenta de lo aprendido al final del proceso, dirían Paulo Freire o Estanislao Zuleta «interesa el producto final».

A pesar de que para los estudiantes el trabajo con apuestas alternativas de evaluación es menor, la posibilidad de hacer una evaluación que vaya indicando el proceso que poco a poco desarrolla el estudiante, le posibilita al maestro fortalecer las dificultades que presenta el educando a medida que encuentre falencias. Sin embargo, solo será posible reducir el riesgo de la incertidumbre en el estudiante en la medida en que el seguimiento no esté marcado por la indicación ni la presión del producto final, sino más bien por la importancia de motivar con las actividades a la indagación, la búsqueda, la reflexión y la consecución de objetivos de aprendizaje. Con la prevalencia de la evaluación tradicional, se evidencia también que son pocos los maestros que movilizan recursos para el desarrollo de la interpretación.

\section{Consideraciones finales sobre los hallazgos expuestos}

En vista de que la enseñanza de las ciencias sociales escolares en estas cuatro instituciones educativas muestran tendencias hacia el mantenimiento de formas de enseñanza tradicionales, y por lo tanto la promoción de actitudes apáticas de los estudiantes hacia el área, es necesario que se siga transformando esta realidad hacia el desarrollo de unas ciencias sociales escolares que promuevan la interpretación y por lo tanto el pensamiento social e histórico, ya lo han señalado tanto Tutiaux como Lucart, al considerar que la falta de interés del estudiante está directamente asociada con la forma en cómo el conocimiento ha sido trasmitido $^{39}$, por lo tanto el desinterés escolar estaría relacionado con la falta de reconocimiento de la naturaleza cognitiva del educando.

Sin pretender invisibilizar el interés por transformar la enseñanza de las ciencias sociales por parte de varios maestros, y el reconocimiento de los estudiantes sobre la pertinencia social que tienen las ciencias sociales en la formación del criterio personal hacia la búsqueda de explicaciones sobre la realidad social, dicha transformación debe ir acompañada de nuevas estrategias y herramientas de trabajo pedagógico. Es necesario que las ciencias sociales les sirvan a los estudiantes como un campo de conocimiento que les permita tener una mirada compleja del mundo en el que viven, lo que implica desarrollar habilidades metacognitivas que no se estimulan si se mantienen las formas tradicionales de enseñanza ${ }^{40}$.

Para Jorn Rüsen, el propósito general de la enseñanza de la historia es el de desarrollar y movilizar el pensamiento histórico ${ }^{41}$, habilidad que solo es posible con la formación de conciencia histórica, entiéndase esta como la capacidad que tiene el estudiante de establecer una relación temporal entre el pasado y el presente, para comprender este último y así proyectar una idea de futuro. Esta definición de Rüsen podría ajustarse a la enseñanza de las

\footnotetext{
39 Tutiaux, «Les qüestions socialment vives, un repte per a la Història i la Geografia escolars». L. Lucart, El fracaso y el desinterés escolar en la escuela primaria (Barcelona: Editorial Gedisa, 1990).

${ }^{40}$ R. Salazar, «La construcción del discurso histórico en estudiantes de ESO: El aula como laboratorio» (tesis de doctorado, Universidad de Barcelona, 2015).

${ }^{41}$ Para Jorn Rusen, pensamiento histórico es la capacidad de interpretar el pasado, condición que es posible cuando se apropia el conocimiento científico de la historia, para Rüsen el pensamiento histórico está directamente relacionado con la narración histórica o capacidad de enunciar contar el conocimiento histórico. Rüsen, Razão histórica... Para Seixas y Morton (2013) el pensamiento histórico es un proceso creativo de interpretación del pasado.
} 
ciencias sociales, porque su definición, ubicada en la función social de la enseñanza de las ciencias sociales, permite que el estudiante se coloque en un lugar geográfico donde construye relaciones en medio de un núcleo cultural en el que se han gestado espacios de discusión y concertación política, de vida económica, de actividades culturales y familiares, etc.

Transitar definitivamente hacia una enseñanza de las ciencias sociales escolares que promuevan el concepto acotado por Rüsen ${ }^{42}$ requiere que el estudiante le encuentre valor en su vida al conocimiento social e histórico, para ello, será clave la promoción del interés, posteriormente debe promoverse en el estudiante el interrogante estableciendo una relación directa entre sus problemas reales y la concordancia de estos con el pasado.

Es importante para el desarrollo de habilidades del pensamiento social e histórico que el estudiante tenga claro conceptos de segundo orden como cambio, permanencia, causa, efecto, tiempo histórico, etc. ${ }^{43}$, que son claves para comprender los fenómenos de transformación social; además que sirven también para el desarrollo de habilidades de pensamiento como la interpretación. La familiarización, claridad y aprendizaje de los conceptos de segundo orden permitirán que sea más clara la contextualización de un contenido específico en clase, así como la interpretación por parte de los estudiantes de las fuentes de información para abordar las indagaciones que estén realizando.

A nivel de las estrategias y herramientas, el profesor de Ciencias Sociales debe inquietarse por trabajar pedagógicamente con las herramientas que a nivel metodológico le brindan los métodos propios de las disciplinas sociales, por ejemplo: el trabajo de campo, la consulta de fuentes primarias, la historia oral, la cartografía social, la sistematización; aprovechando una variedad de recursos como las herramientas tecnológicas y la red desde donde se puede acceder a información de archivos históricos, estadísticas, museos virtuales, o para registrar las actividades realizadas como entrevistas o salidas de campo. De igual forma, se pueden utilizar las visitas de campo a diferentes lugares, los documentales, el cine, la música, entre otros, que aterrizados asertivamente en el ejercicio pedagógico pueden movilizar los métodos propios de las disciplinas sociales hacia el desarrollo de pensamiento social e histórico con los estudiantes.

Los hallazgos de la investigación arrojan coincidencias entre lo referido por los profesores y los estudiantes que afianzan la idea de la confluencia entre viejos paradigmas y nuevas realidades. Estos agentes concuerdan en que las herramientas más usadas son el tablero, el marcador y el libro de texto; en contraste, aunque no menos importante, pero sí con menor incidencia, se encuentra el uso del cine, los documentales, las computadoras, esto evidencia de parte del profesor una búsqueda de equilibrios entre la hegemonía de la clase magistral centrada en el discurso y la intención de dinamizar y hacer más experiencial la enseñanza de los procesos sociales, espaciales e históricos.

En definitiva, debe transitarse a una concepción nueva de las ciencias sociales escolares, en donde aprender sobre la realidad histórica y social debe ser el resultado de un proceso de interrogación y descubrimiento orientado por el maestro, caracterizado por la provocación, la indagación, la organización de fuentes de información y la interpretación ${ }^{44}$. Se debe procurar que en este proceso el estudiante adquiera la capacidad de comprensión e

\footnotetext{
${ }^{42}$ Rüsen, Razão histórica...

${ }^{43}$ Seixas y Peck, «Teaching historical thinking».

${ }^{44}$ J. Rüsen, «¿Puede mejorar el ayer? Sobre la transformación del pasado en historia», en Política, identidad y narración, coord. por Gustavo Leyva (Iztapalapa: Universidad Autónoma Metropolitana, 2003), 477-501.
} 
interpretación ${ }^{45}$; es decir, que logre entender y dar cuenta de manera crítica sobre la realidad social e histórica estudiada, buscando responder desde su criterio a las posibles problemáticas que su entorno y su sociedad esté enfrentando, para así proyectar una idea de futuro ${ }^{46}$.

La investigación concluye que las razones que motivan al desinterés por el área es propio de una enseñanza centrada en contenidos predefinidos, de la monotonía que se da en el desarrollo habitual de las clases, de la irrelevancia que llega a tener en los estudiantes los contenidos predefinidos, del uso de estrategias y herramientas que promueven la reproducción de información que está consignada en los libros de texto y del mantenimiento de la evaluación final escrita como un ejercicio que mide el estudiante en términos de producto final y no como parte de un proceso formativo.

\section{Referencias}

Arboleda, J. Competencias pedagógicas: conceptos y estrategias. Bogotá: Editorial REDIPE, 2011.

Avanzini, G. «La incertidumbre de la evaluación». En La pedagogía hoy, editado por G. Avanzini. México: Fondo de Cultura Económica, 1998.

Betancur, L.B. «Una mirada a las prácticas de enseñanza de las ciencias sociales en Guadalajara de Buga». Revista de investigaciones. Universidad Católica de Manizales 16, n. 2 (2016).

Betancur, L.B. «A propósito de Bauman y los retos de la educación en la modernidad liquida: cavilaciones sobre el futuro de la enseñanza de la Historia». En El prisma de la formación docente en Colombia: teoría pedagógica y experiencias didácticas, editado por C. Cano, 27-57. Cali: Editorial Universidad Santiago de Cali, 2017.

Buitrago, B. «La didáctica: un acontecimiento vivo en el aula». Revista Científica Guillermo de Ockham 6, n. 2 (2008).

Carretero, M. Documentos de identidad: la construcción de la memoria histórica en un mundo global. Buenos Aires: Paidós, 2007.

Congreso de la República de Colombia. Ley 115 del 8 de febrero de 1994, ley general de educación. https://www.mineducacion.gov.co/1621/articles-85906_archivo_pdf.pdf

Foucault, M. Microfísica del poder. Madrid: Ediciones de la Piqueta, 1979.

\footnotetext{
${ }^{45}$ Arboleda, Competencias pedagógicas...

${ }^{46}$ Rüsen, Razão histórica...
} 
Guerrero, C. «La incidencia de las reformas educativas en la enseñanza de la historia en Colombia, 1973-2007». Tesis de maestría. Universidad Nacional de Colombia, Bogotá, 2011.

Ghotme, R. «La identidad nacional, el sistema educativo y la historia en Colombia 19101962». Revista Científica General José María Córdova 11, n. 11 (2013): 273-289.

González. M. «La configuración histórica del saber pedagógico para la enseñanza de la historia en Colombia, trazos de un camino: 1870-2010». Tesis de maestría, 2011.

\section{https://www.javeriana.edu.co/biblos/tesis/csociales/tesis249.pdf}

Lucart, L. El fracaso y el desinterés escolar en la escuela primaria. Barcelona: Editorial Gedisa, 1990.

McMillan, J. Investigación Educativa. Madrid: Pearson Educación S.A., 2005.

Mosquera, S., G. Villa y A. M. Riascos. «Interrelación de los sistemas (político, económico, educativo y religioso) y su afectación en el subsistema de la enseñanza de la historia de Colombia en el periodo de 1900 a 1958». Trabajo de grado, Universidad Santiago de Cali, Cali, 2017.

Pagès, J. «Enseñar y aprender ciencias sociales en el siglo XXI: reflexiones casi al final de una década». Investigación en Educación, Pedagogía y Formación Docente, II Congreso Internacional, Medellín, 2009.

- «Ciudadanía global y enseñanza de las Ciencias Sociales: retos y posibilidades para el futuro». Revista de Investigación en Didáctica de las Ciencias Sociales, REIDICS, n. 5 (2019): 5-22. doi: https://doi.org/10.17398/2531-0968.05.5

Quiceno, H. Crónicas históricas de la educación en Colombia. Bogotá: Cooperativa Editorial Magisterio, 2003.

Rodríguez, N. Neuroeducación para padres. Barcelona: Grupo Z, 2016.

Ruiz, M. «La competencia estratégica del docente ante la violencia escolar en países en vías de desarrollo». Revista Interuniversitaria de Formación del Profesorado n. ${ }^{\circ} 45$ (2002): 169-182.

Rüsen, J. Razão histórica: Teoría da historia, fundamentos da ciência histórica. Brasilia: Editora Universidad de Brasilia, 2001. 
- «Puede mejorar el ayer? Sobre la transformación del pasado en historia». En Política, identidad y narración, coordinado por Gustavo Leyva, 477-501. Iztapalapa: Universidad Autónoma Metropolitana, 2003.

Sacristán, J. El curriculum: una reflexión sobre la práctica. Madrid: Ediciones Morata, 2007.

- «Diez tesis sobre la aparente utilidad de las competencias». En Educar por competencias ¿Qué hay de nuevo?, editado por J. Sacristán. Madrid: Ediciones Morata, 2008.

Salazar, R. «La construcción del discurso histórico en estudiantes de ESO: El aula como laboratorio». Tesis de doctorado, Universidad de Barcelona, 2015.

Santisteban, A. «Las finalidades de la enseñanza de las Ciencias Sociales». En Didáctica del conocimiento del medio social y cultural en la educación primaria, editado por J. Pagès y A. Santisteban. Madrid: Editorial Síntesis, 2016.

«La enseñanza de las Ciencias Sociales a partir de problemas sociales o temas controvertidos: estado de la cuestión y resultados de una investigación». El Futuro del Pasado 10 (2019): 57-79. doi: http://dx.doi.org/10.14516/fdp.2019.010.001.002

Sanz, G. Comunicación efectiva en el aula. España: Editorial Grao, 2005.

Saldarriaga, O. «Del oficio de maestro ¿de intelectual subordinado a experto subordinador?». Revista Educación y Ciudad, IDEP, n. 11 (2006): 53-70.

Seixas, P. y C. Peck. «Teaching historical thinking». En Challenges and Prospects for Canadian Social Studies, editado por A. Sears y I. Wright, 109-117. Vancouver: Pacific Educational Press, 2004.

Seixas, P. y T. Morton. The Big Six Historical Thinking Concepts. Toronto: Nelson Education Ltd, 2013.

Tutiaux, N. «Les qüestions socialment vives, un repte per a la Història i la Geografia escolars». En Les qüestions socialment vives i l'ensenyament de les ciències socials, editado por J. Pagès y A. Santisteban, 25-44. Barcelona: Universidad Autónoma de Barcelona, 2011.

Voss. J.F., J. Wiley y J. Kennet. J. «Las percepciones de los estudiantes acerca de la historia y los conceptos históricos». En Aprender y pensar la historia, editado por M. Carretero y J. Voss, 295- 329. Buenos Aires: Amorrortu, 2004.

Zambrano, A. Didáctica, pedagogía y saber. Bogotá: Cooperativa Editorial Magisterio, 2005. 